\title{
A novel heterozygous mutation in the HMBS gene in a patient with acute intermittent porphyria and posterior reversible encephalopathy syndrome
}

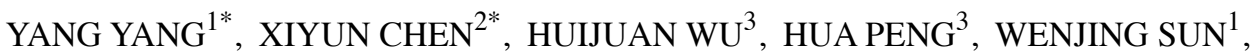 \\ $\mathrm{BIN} \mathrm{HE}^{3}$ and ZHENGANG YUAN ${ }^{2}$
}

\begin{abstract}
Departments of ${ }^{1}$ Neurology and ${ }^{2}$ Hematology and Oncology, The Third Affiliated Hospital of
The Second Military Medical University, Shanghai 200438; ${ }^{3}$ Department of Neurology, The Second

Affiliated Hospital of The Second Military Medical University, Shanghai 200003, P.R. China
\end{abstract}

Received September 18, 2019; Accepted April 4, 2020

DOI: $10.3892 / \mathrm{mmr} .2020 .11117$

\begin{abstract}
Acute intermittent porphyria (AIP) is a rare inherited disorder, which is caused by the partial deficiency of hydroxymethylbilane synthase (HMBS), an enzyme of the heme biosynthetic pathway. Abdominal pain, neuropsychiatric disturbance and neuropathy are the typical manifestations of the disease. Complications such as posterior reversible encephalopathy syndrome (PRES), a rare type of brain lesion present on MRI, are also observed in patients with AIP. The present study reports on the case of a 36-year-old Chinese female patient with AIP and PRES. Genomic DNA were obtained from peripheral blood leukocytes and genomic regions of the HMBS gene were amplified as 2 fragments, which together contained all the exons and flanking intronic regions. Sanger sequencing of the amplified DNA fragments from the patient and the patient's family revealed a novel frameshift deletion (c.405-406delAA) in exon 8 of the HMBS gene. This mutation leads to a subsequent truncated protein (p.Glu135AspfsX74). The recombinant mutant protein had $62 \%$ activity relative to the wild-type protein but similar thermostability. It was confirmed that this novel mutation was the cause of AIP. Accumulation of D-aminolevulinic acid
\end{abstract}

Correspondence to: Professor Zhengang Yuan, Department of Hematology and Oncology, The Third Affiliated Hospital of The Second Military Medical University, 700 Moyu North Road, Jiading, Shanghai 200438, P.R. China

E-mail: yuanzg@163.com

Professor Bin He, Department of Neurology, The Second Affiliated Hospital of The Second Military Medical University, 415 Fengyang Road, Huangpu, Shanghai 200003, P.R. China

E-mail: binhe97@163.com

*Contributed equally

Key words: acute intermittent porphyria, hydroxymethylbilane synthase, mutation, posterior reversible encephalopathy syndrome
(ALA) due to HMBS dysfunction is a potential mechanism of PRES. The manifestation of PRES may be associated with ALA-induced cytotoxicity and the destruction of the blood-brain barrier. In summary, in the present study, a novel pathogenic HMBS mutation was identified, expanding on the molecular heterogeneity of AIP.

\section{Introduction}

Porphyrias is a group of rare, mostly inherited disorders that are each caused by a defect in a specific heme biosynthetic enzyme (1). Acute intermittent porphyria [AIP; Online Mendelian Inheritance in Man (OMIM) ID, 176000] is caused by the partial deficiency of hydroxymethylbilane synthase (HMBS; EC:4.3.1.8), an enzyme in the heme biosynthetic pathway (2). Recent updates for the management of acute attacks of porphyria have described the clinical features of an AIP episode as including abdominal pain, nausea, vomiting, constipation, dark urine, hypertension, arrhythmia, psychosis, convulsions, peripheral motor neuropathy and hyponatremia (3). Abdominal pain, psychiatric disturbance and peripheral neuropathies are the 'classical triad' of AIP (4).

With the advent of DNA technology, genetic analysis has become the gold standard for the diagnosis of AIP (2). In total, $>390$ different mutations responsible for AIP have been reported so far (1), most of them in Europe, particularly in northern Europe (5).

At our department, a Chinese patient with AIP and posterior reversible encephalopathy syndrome (PRES) was identified. PRES is characterized by several neurological symptoms typically corresponding to areas of vasogenic cerebral edema on MRI (6).

The patient of the present study was diagnosed with AIP by genetic analysis and clinical presentation of PRES based on typical symptoms and imaging results. PCR-based gene sequencing identified a frameshift deletion (c.405-406delAA) in exon 8 of the HMBS gene. Furthermore, the deletion variant truncated (p.Glu135AspfsX74) the HMBS protein in the proband. 


\section{Materials and methods}

Sample collection. All procedures were performed in accordance with the ethical standards of the responsible Ethics committee of The Second Military Medical University (Shanghai, China) on human experimentation and with The Declaration of Helsinki from 1975 and its revision from 2000. Blood samples were collected in EDTA from a 36-year-old Chinese female in May 2013, who was referred by her physicians due to elevated D-aminolevulinic acid (ALA) and porphobilinogen (PBG) levels, and due to having clinical symptoms compatible with acute hepatic porphyria. The patient provided informed consent for genetic testing and the study was approved by the Ethics Committee of The Second Military Medical University (Shanghai, China).

DNA isolation and mutation analyses. Genomic DNA was extracted from lymphoblasts using the QIAamp DNA Blood Mini kit (Qiagen, Inc.). Amplification of the entire HMBS gene was performed in two parts as described previously (7). Fragment 1 comprised the promoter region through intron 3 [primers long-range (LR)1 and LR2] and fragment 2 was comprised of exon 2 through exon 15 (primers LR3 and LR4), as presented in Table I. Using the Extensor ${ }^{\mathrm{TM}} \mathrm{Hi}$-fidelity PCR master mix (Thermo Fisher Scientific, Inc.), initial denaturation was performed at $94^{\circ} \mathrm{C}$ for $1 \mathrm{~min}$. For the first 16 cycles, denaturation was performed at $94^{\circ} \mathrm{C}$ for $30 \mathrm{sec}$, with annealing and extension at $67^{\circ} \mathrm{C}$ for $5 \mathrm{~min}$. The next 12 cycles included denaturation at $94^{\circ} \mathrm{C}$ for $30 \mathrm{sec}$, and annealing and extension at $67^{\circ} \mathrm{C}$ for $5 \mathrm{~min}$ and $15 \mathrm{sec}$, respectively, with 15 -sec increments at each additional cycle. The final extension was performed at $72^{\circ} \mathrm{C}$ for $10 \mathrm{~min}$. PCR products were analyzed by agarose gel electrophoresis to determine that the long-range reactions were successful and to identify any gross gene rearrangements.

The amplified fragments were sequenced on the ABI 3500 Genetic Analyzer (Thermo Fisher Scientific, Inc.) according to the manufacturer's protocol. A list of the sense and antisense primers used and sequencing parameters are provided in Table I. In brief, sequencing PCR was performed as follows, following the manufacturer's instructions: Denaturation at $95^{\circ} \mathrm{C}$ for $30 \mathrm{sec}$; annealing at $60^{\circ} \mathrm{C}$ for $30 \mathrm{sec}$; and extension at $72^{\circ} \mathrm{C}$ for $60 \mathrm{sec}$ for 30 cycles. After a final denaturation for $3 \mathrm{~min}$ at $94^{\circ} \mathrm{C}$, the PCR mixture was loaded on a $2 \%$ agarose gel and air-dried for $15 \mathrm{~min}$. The PCR product was excised from the gel, purified with a QIAEX II gel extraction kit (Qiagen, Inc.) and then subjected to capillary electrophoresis sequencing using the ABI 3500 Genetic Analyzer (Thermo Fisher Scientific, Inc.).

Generation and expression of $H M B S$ constructs in $E$. coli. The full-length human wild-type (WT) and mutant (mut; c.405-406delAA) the synthesized HMBS complementary DNA (Coding sequence of HMBS was download from NCBI database, https://www.ncbi.nlm.nih. gov/nuccore/NM_001024382.2, and cDNA was syntehsised by reverse transcription-PCR.) (8) was cloned into the PKK233-3 vector (Ybscience, Inc.; http://www.ybio.net/) and designated as PKK-WT $(1.743 \pm 0.047 \mathrm{mg} / \mathrm{ml})$ and PKK-mut $(1.642 \pm 0.168 \mathrm{mg} / \mathrm{ml})$, respectively. The constructs were confirmed by sequencing and transformed into BL21 E. coli competent cells (Ybscience, Inc.), which were cultured overnight in lysogeny broth medium (cat. no. ST156; Beyotime Institute of Biotechnology) at $37^{\circ} \mathrm{C}$. Inoculates were induced with $1 \mu \mathrm{m}$ isopropyl $\beta$-D-1-thiogalactopyranoside and incubated for an additional $3 \mathrm{~h}$. at $37^{\circ} \mathrm{C}$ After centrifugation at $6,000 \mathrm{x} \mathrm{g}$ for $1 \mathrm{~min}$ at room temperature, cells were resuspended in $1 \mathrm{ml}$ bacterial lysis buffer (cat. no. C500003; Sangon Biotech Co., Ltd.) and lysed by freezing at $-80^{\circ} \mathrm{C}$ for $30 \mathrm{~min}$, followed by thawing. Lysates were centrifuged at $12,000 \times \mathrm{g}$ for $5 \mathrm{~min}$ at $4^{\circ} \mathrm{C}$, and supernatants were collected and stored at $-80^{\circ} \mathrm{C}$ in the dark until further use. The time interval between transfection and subsequent experiments was $<1$ week.

Western blot analysis. Denatured protein lysates were separated by $8 \%$ SDS-PAGE and transferred on to a polyvinylidene difluoride membrane (Beyotime Institute of Biotechnology). The lane of PKK223-1-1 contained $0.52 \mathrm{mg}$ protein. The lane of PKK223-1-2 contained $0.56 \mathrm{mg}$ protein. The lane of PKK223-1-3 contained 0.62 mg protein. The lane of HMBS mut-1-1 contained $0.46 \mathrm{mg}$ protein. The lane of HMBS mut-1-2 contained $0.58 \mathrm{mg}$ protein. The lane of HMBS mut-1-3 contained $0.48 \mathrm{mg}$ protein. The lane of HMBS WT-5-1 contained $0.48 \mathrm{mg}$ protein. The lane of HMBS WT-5-2 contained $0.50 \mathrm{mg}$ protein. The lane of HMBS WT-5-3 contained $0.56 \mathrm{mg}$ protein. Determination of protein concentration using bicinchoninic acid Protein Assay kit (cat. no. P0012s; Beyotime Institute of Biotechnology). After blocking in 5\% non-fat dry milk in Tris-buffered saline containing Tween-20 for $3 \mathrm{~h}$ at room temperature, the membrane was incubated with anti-HMBS antibody (1:5,000; cat. no. ab129092; Abcam) at $4^{\circ} \mathrm{C}$ overnight, followed by incubation with secondary horseradish peroxidase (HRP)-conjugated anti-rabbit IgG (1:5,000; cat. no. Ab6721; Abcam) for $2 \mathrm{~h}$ at room temperature. Signals were detected using the ECL Chemiluminescence substrate reagent kit (Invitrogen; Thermo Fisher Scientific, Inc.). Membranes were stripped and re-probed with anti-GAPDH $(1: 1,000$; cat. no. AG019-1; Beyotime Institute of Biotechnology) and secondary HRP-conjugated anti-mouse $\operatorname{IgG}$ (1:5,000; cat. no. A0261; Abcam) at room temperature for $1 \mathrm{~h}$ for loading normalization.

HMBS enzyme activity and thermostability. HMBS enzyme activity was determined in lysates by measuring the conversion of PBG to uroporphyrin as previously described (9). Lysates were diluted 1:3 in phosphate buffer $(\mathrm{pH} 7.6)$ containing dithiothreitol, $\mathrm{MgCl}_{2}$ and Triton $\mathrm{X}-100 ; 100 \mu \mathrm{l}$ of this mixture was pre-incubated with $1.8 \mathrm{ml}$ of $0.1 \mathrm{M}$ Tris- $\mathrm{HCl}$ (pH 8.1) for $3 \mathrm{~min}$ at $37^{\circ} \mathrm{C}$, followed by incubation with $0.5 \mathrm{ml}$ of $1 \mathrm{~mm}$ PBG substrate for $60 \mathrm{~min}$ at $37^{\circ} \mathrm{C}$ in the dark. The reaction was stopped with $350 \mu$ l cold $40 \%$ trichloroacetic acid and oxidation of uroporphyrinogen to uroporphyrin was performed under sunlight for $30 \mathrm{~min}$. Uroporphyrins were measured quantitatively by spectrofluorometry at an emission wavelength of $405 \mathrm{~nm}$. HMBS activity was expressed in the units pmol uroporphyrin/mg protein/h using appropriate standards. All assays were performed in triplicate and average values were expressed as percent of WT activity. For enzyme thermostability studies, WT and mutant lysates were assayed for HMBS activity after incubation at $65^{\circ} \mathrm{C}$ for $90 \mathrm{~min}$. 
Table I. Primers for PCR amplification and sequencing.

Primer

Oligonucleotide sequence (5'-3')

\begin{tabular}{|c|c|}
\hline \multicolumn{2}{|c|}{ For long-range PCR } \\
\hline LR1 (sense) & TGCTCCCACTTCAGTTACTTGTCTTTA \\
\hline LR2 (antisense) & GACGCCCATCTCTAАACCTAATCAGG \\
\hline LR3 (sense) & AAGGGACCAGCCTTGGAGTATTTCCC \\
\hline LR4 (antisense) & CAAGGATAGAAGGGCGGTTGAGGTG \\
\hline \multicolumn{2}{|c|}{ For direct sequencing } \\
\hline Exon 1 & GAGACCAGGAGTCAGACTGT \\
\hline Exon $2 / 3$ & CCCACTGACAACTGCCTTGGTCAAG \\
\hline Exon 4 & CCTAACCTGTGACAGTCT \\
\hline Exon $5 / 6$ & AGACCTAGCATACTAGGG \\
\hline Exon 7 & AGGGTCAGGCCCCAAAGGGAAAGG \\
\hline Exon 8 & CGAGAGAATAGAGGTGAT \\
\hline Exon 9 & TTGTCTTTTTCCTTGGCTGC \\
\hline Exon 10 & GGGAAAGACAGACTCAGGCAGAG \\
\hline Exon 11 & CGGTAGCATCCCAAGGTCT \\
\hline Exon 12 & TAAGAAATCTTCCCTGC \\
\hline Exon 13 & CAGTGATGTCCTCAGGTCTG \\
\hline Exon 14 & ATCCCAGGTTTCTAGGTAG \\
\hline \multirow[t]{2}{*}{ Exon 15} & AGACCATGCAGGCTACCATC \\
\hline & CGTGACCTGTCGTCGTTG \\
\hline
\end{tabular}

LR, long range.

Mapping of the missense mutation on the human HMBS crystal structure. The 2.8- $\AA$ crystal structure of human HMBS (10) (Protein Data Bank ID: 3EQ1) was viewed in PyMOL Molecular Graphics System (v2.0; Schrödinger, LLC). Mapping of the molecular mutations and graphical representations were created on PyMOL Molecular Graphics System (v2.0; Schrödinger, LLC) (10), which involved comparing the structure of a protein simulated by the software with that of a published protein.

Statistical analysis. Independent-samples t-tests were used as appropriate to compare the thermostability between WT-HMBS and mutant protein. The ratio of the enzyme activity prior to and after heating was used as an index to describe the thermostability of HMBS. SPSS version 21.0 (IBM Corp.) was used for analysis. $\mathrm{P}<0.05$ was considered to indicate statistical significance.

\section{Results}

Case report. The proband was a 36-year-old Chinese female. She was treated at a local county hospital for severe acute abdominal pain five years previously, for which she underwent a laparotomy for intussusception, but the results were misdiagnoses. She then had a recurrence of acute lower abdominal pain accompanied by systemic seizures six months later; she recovered after one month of conservative treatment. The acute abdominal pain recurred two years ago, and after one week of this persistent pain, the patient developed neurological and psychiatric symptoms, including blurred vision, impaired speech, seizures and memory impairment. At one month after the onset, the patient was referred to the Neurology Department of The Second Affiliated Hospital of The Second Military Medical University for further diagnosis. Medical examination indicated that the patient's blood pressure was normal with good heart and lung functions. Neurological examination revealed mental fatigue and limb paralysis. Brain MRI indicated intracranial multifocal lesions in the cortical and subcortical white matter of the left temporal, parietal, occipital and right occipital lobes. After two weeks of treatment, MRI revealed partial regression of the neurological abnormalities (Fig. 1). Laboratory tests indicated that the patient had hypoproteinemia (25 g/l), hypoglycemia and mildly elevated alanine aminotransferase and aspartate aminotransferase, while levels of serum ceruloplasmin and blood electrolytes (potassium, sodium, chloride, calcium and magnesium ions) were in the normal range. An electroencephalogram revealed slower brain waves in the bilateral cerebral hemispheres but no epileptic discharge. Chest X-rays were normal. Pelvic ultrasound revealed intrauterine pregnancy, which was the cause of changes in hormone levels. PBG was strongly positive in the qualitative test (Watson-Schwartz test) $(11,12)$ of the patient's urine during the attack period, which became negative after the episode. Repeated tests performed during the remission period were weakly positive for PBG. Darkening of urine after 30 min of exposure to sunlight confirmed the clinical diagnosis of AIP. The patient had high levels of porphyrin precursors in her urine. The proband was treated by intravenous infusion of $300 \mathrm{~g} /$ day of glucose. Complete recovery was achieved after 2 weeks of conservative treatment, and then the patient chose to terminate the pregnancy. 
A

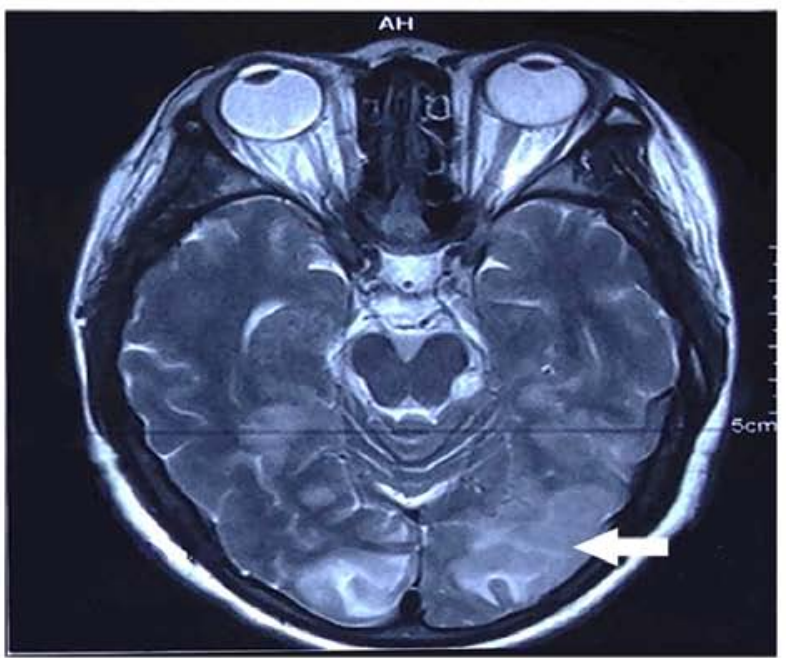

C

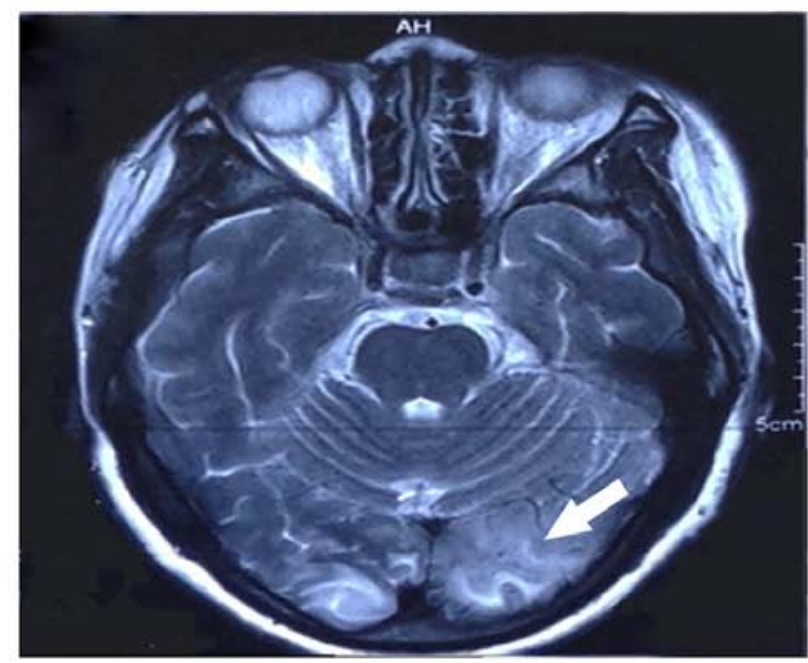

E

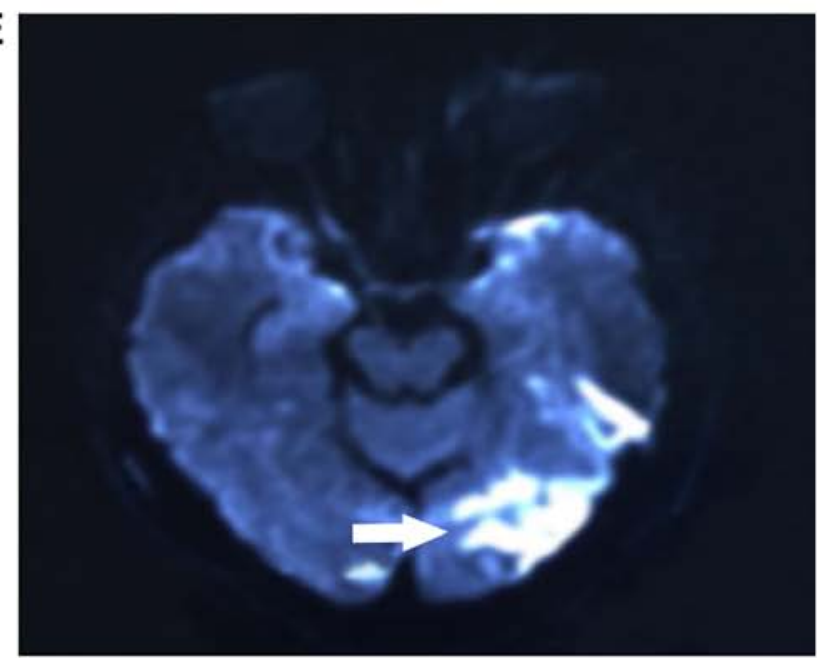

B

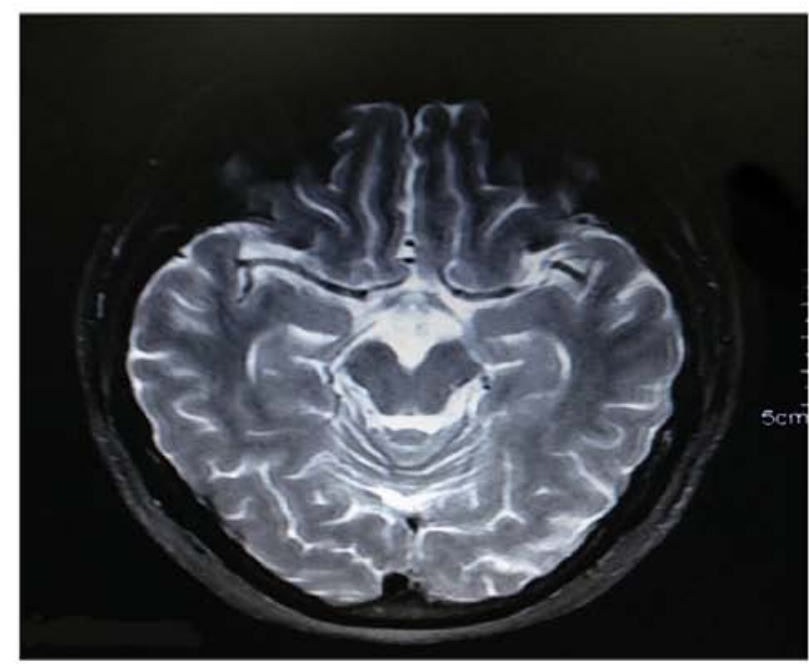

D

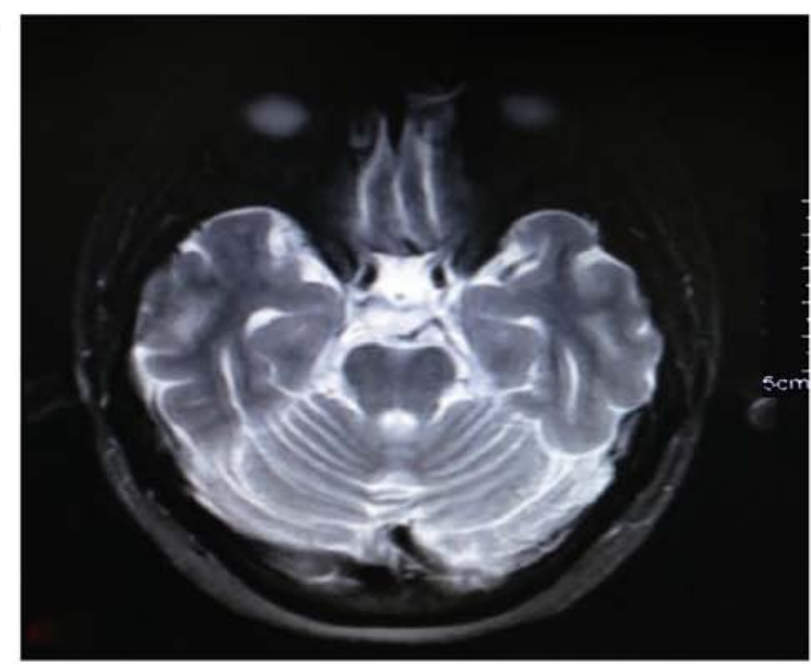

F

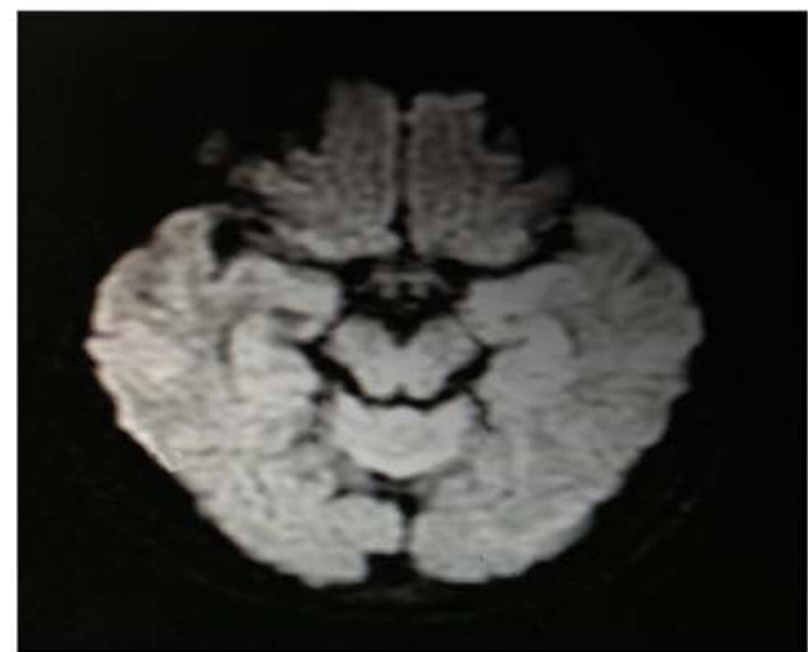

Figure 1. MRI images of the patient. (A) Prior to treatment, T2 signal abnormalities were observed in the bilateral occipital lobe. (B) These abnormal signals were almost recovered and returned to normal after treatment with 1 week. (C) Prior to treatment, T2 signal abnormalities were observed in the bilateral occipital lobe. (D) These abnormal signals were almost recovered and returned to normal after treatment with 1 week. (E) Diffusion-weighted image signal abnormalities were observed in the same regions, suggestive of vasogenic edema and compatible with posterior reversible encephalopathy syndrome; white arrow indicates diseased regions. (F) These abnormal signals were almost recovered and returned to normal after treatment with 1 week.

For genetic screening, blood samples of the patient and her family members (the patient's parents and the patient's son) were collected after informed consent. The patient's mother developed symptoms of psychosis during her middle age and was diagnosed with schizophrenia, which was managed with antipsychotic drug treatment but was not diagnosed with 


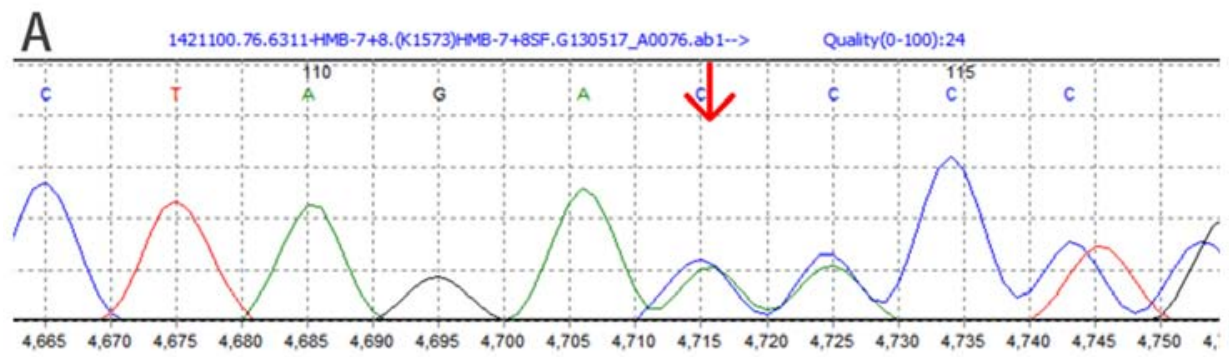

B $\quad$ 14322242.47.6313+MB -7+8.(K1945)HW-7+8SF.61305254338.ab1-> Quality(0-100):21

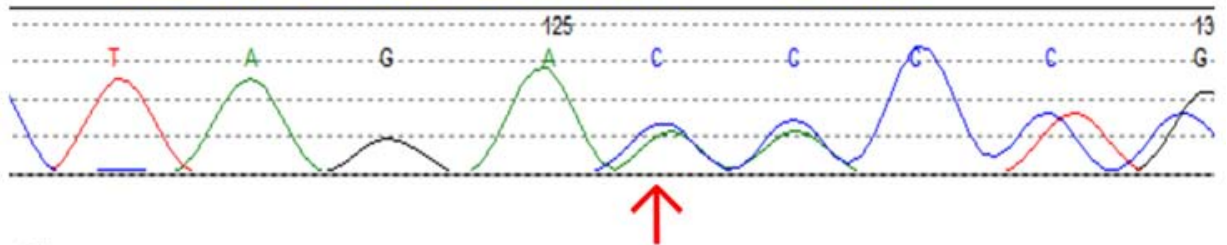

C 1432242.49.6314HMB-7+8.(K1945)HMB-7+8SF.G1305254390.ab1-> Quality(0-100):14

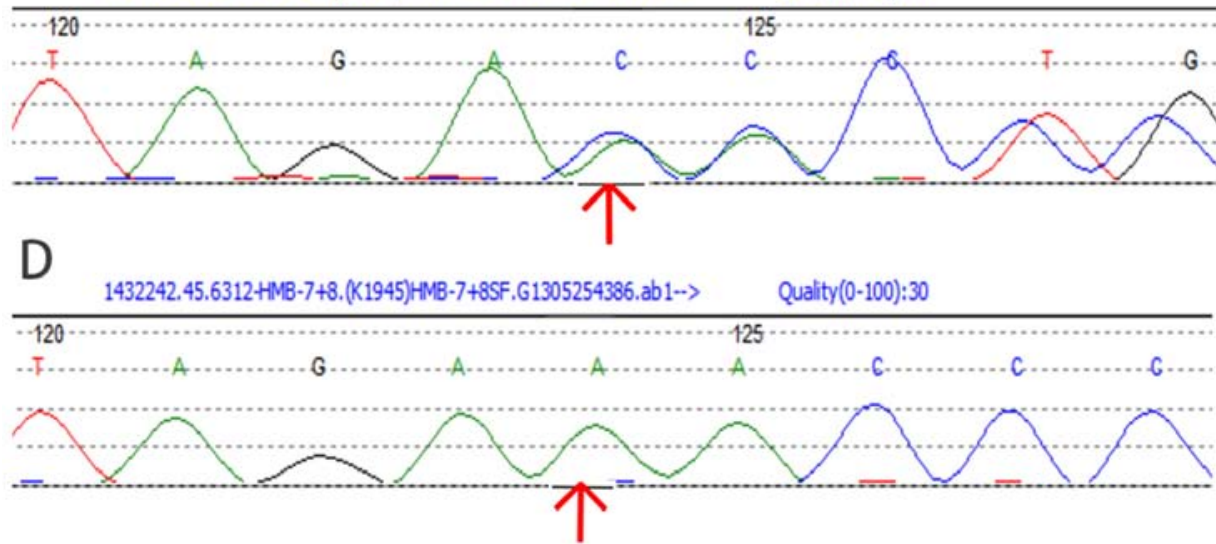

Figure 2. Mutation sequence of HMBS of the patient and members of the patient's family. DNA sequencing chromatogram of exon 8 of HMBS transcripts (red arrow) from (A) the patient, (B) the patient's mother and (C) the patient's son, heterozygous for the c.405-406delAA mutation. (D) The sequence from the patient's father is normal. HMBS, hydroxymethylbilane synthase.

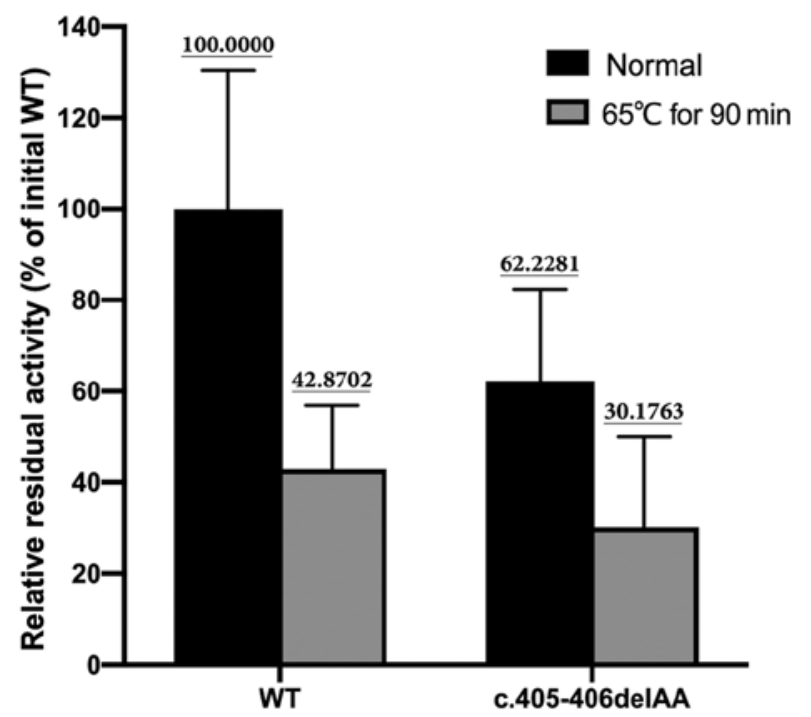

Figure 3. Mutant protein is thermally stable. The thermostability of the WT and mutation enzymes was assessed by comparing their respective enzyme activity with the initial WT activity prior to thermoinactivation. The experimental results reflect the means of four independent experiments and the error bars denote 1 standard deviation. WT, wild-type.
AIP. The patient's son (age, 18 years) had not developed any abdominal pain or nervous system dysfunction.

Mutation analysis of the HMBS gene. Direct sequencing of PCR-amplified genomic DNA, including 15 exons and adjacent intronic areas, identified a c.405-406delAA frameshift mutation in exon 8 of the HMBS gene (Fig. 2). This was a heterozygous mutation, identified in the proband, the proband's mother and the proband's son, but not in the proband's father.

Enzyme activity and thermostability of the mutated HMBS. HMBS enzyme activity of the mutant protein was $62.2 \%$ of the activity of the expressed WT protein $(71,449.00 \pm 21,708.96$ vs. $44,461.00 \pm 14,412.49 \mathrm{U}, \mathrm{t}=1.794, \mathrm{P}=0.147)$. The thermostability assay indicated that the mutant protein retained $48.5 \%$ of its initial HMBS activity after incubation at $65^{\circ} \mathrm{C}$ for $90 \mathrm{~min}$. On the other hand, the WT enzyme retained $42.9 \%$ of its initial activity after a 90 -min incubation at $65^{\circ} \mathrm{C}$ (Fig. 3), and there was no statistically significant difference in thermostability between the WT protein and mutated protein ( 42.9 vs. $48.5 \%$; $\mathrm{P}=0.703$ ). 


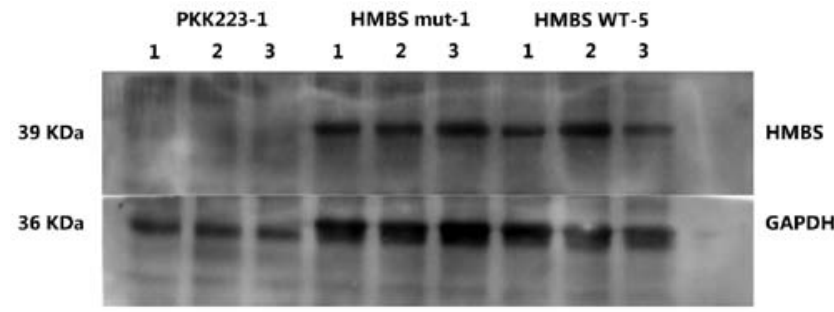

Figure 4. Western blot analysis of HMBS deletion mutants. The stability of the expressed mutant proteins was compared with that of the WT by western blot analysis. HMBS, hydroxymethylbilane synthase; mut, mutant; WT, wild-type.

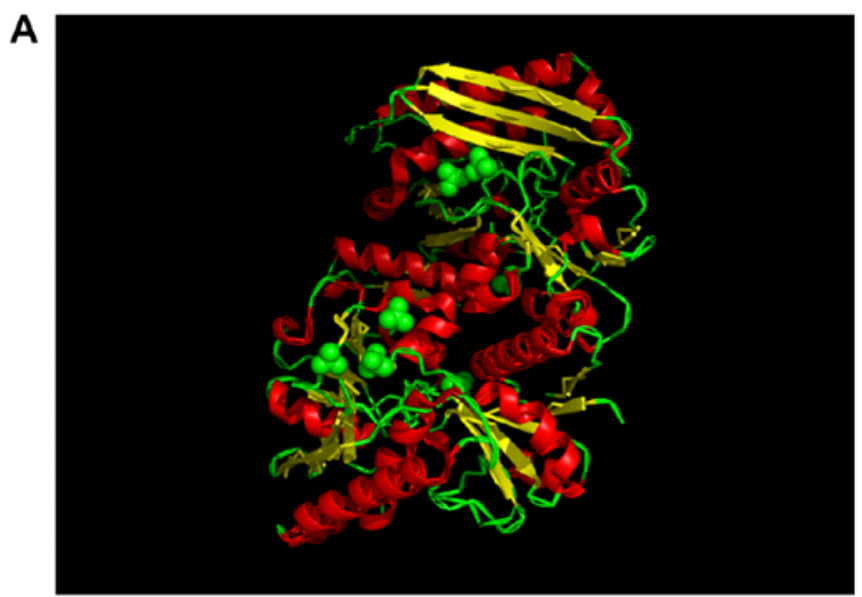

B

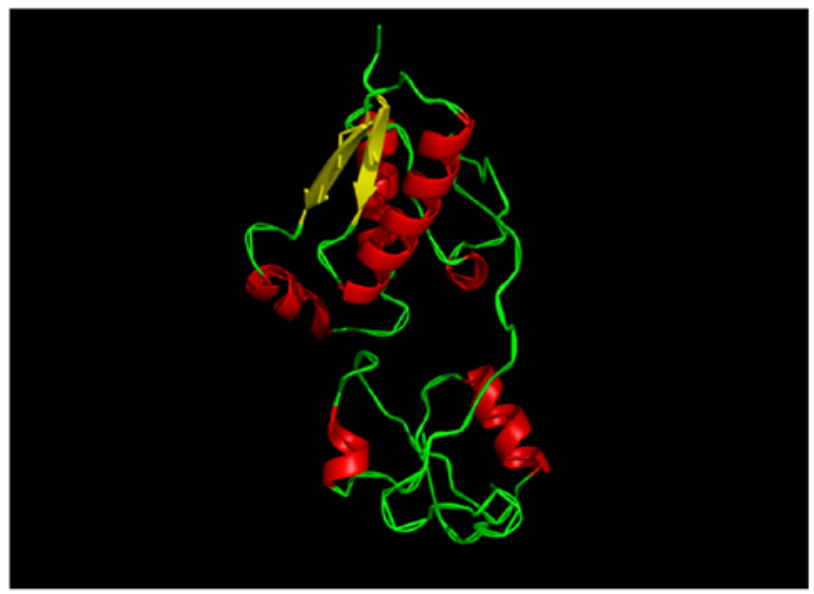

Figure 5. Mapping of the deletion mutation on the structure of the human hydroxymethylbilane synthase protein. (A) An image of the normal structure (B) An image of the mutant structure.

Effects of deletion mutation on protein stability and structure of the mutant HMBS protein. Western blot analysis indicated that the expression of the mutant protein (Glu135AspfsX74) was similar to that of the WT protein (Fig. 4). However, the secondary structure of the truncated protein was notably different from that of the WT. The mutant protein exhibited a significant reduction in $\alpha$-helix and $\beta$-sheets and folding of the peptide chains (Fig. 5).

\section{Discussion}

AIP is an inherited metabolic disease characterized by the partial deficiency of HMBS (1). In the present study, a novel frameshift deletion (c.405-406delAA) in exon 8 of the HMBS gene leading to a truncated (p.Glu135AspfsX74) protein was identified in a Chinese pedigree (Fig. 6). The structure of the mutated protein was significantly different from the WT and its enzyme activity was lower than that of the WT protein with similar thermostability. As no previous records of the mutation were present in the OMIM database, it was inferred that this was a novel mutation identified in a Chinese family.

As exemplified in the present case, AIP is easily and frequently misdiagnosed in the clinic (1). In spite of the presentation with clinical symptoms of AIP, including acute abdominal pain, neuropathy and psychiatric disorders, the patient of the present study underwent laparotomy for intussusceptions. Following the onset of the recent episode of disease activity, AIP was diagnosed based on the typical clinical manifestations, urine PBG test and genetic testing.

In humans, HMBS is the third enzyme of the heme biosynthetic pathway that has a high degree of conservation (2). Human HMBS consists of three domains: Domains 1 and 2 have five $b$-sheets and three a-helices; and domain 3 has a flattened b-sheet geometry. The functional core of HMBS enzyme activity is located in domain 1 (13). It has the DPM cofactor in the native reduced conformation with an ordered sulphate ion hydrogen bound to $\mathrm{Arg}^{26}$ and $\mathrm{Ser}^{28}$ at the proposed substrate-binding site (13). Although domain 3 is distant from domain 1, its interaction with domain 1 may modulate conformational fluctuations associated with the enzyme action (13). In the present study, mutations led to the truncation of the HMBS protein. It is worth noting that western blot analysis demonstrated that the truncated mutant proteins had similar molecular weights to those of WT proteins. It was demonstrated that the mutation was the deletion of two bases $\mathrm{AA}$, and after the base deletion, the primary structure of the protein changed. The translation was terminated prematurely, but it was not possible to determine what changes occurred in the tertiary structure of the protein, and thus no significant difference was observed in the western blotting results. However, the present study was unable to verify the protein structure changes. Therefore, the structural changes caused by this mutation will be investigated in further studies. The mutant protein is p.Glu135AspfsX74. This means that the missing AA resulted in the conversion of amino acid residues at position 135 from Glu to Asp, and premature termination occurred at position 74 after this position. ACC is not a stop codon so this mutation results in frameshift mutations and produces different amino acid residues in subsequent sequences. According to the prediction analysis of the truncated protein, the mutant protein has 300 amino acid residues. Compared to the WT protein, the mutant protein does lose $\sim 60$ residues, primarily affecting the domain 3 regions. However, structural prediction revealed that the b-folding and the a-coiling of the mutant protein were reduced, which may be due to the loss of domain 3 affecting the correct folding of the domain 1 and 2 region peptide chains. It was concluded that the function of the mutated protein was reduced to $\sim 60 \%$ of the WT protein because the functional core of the domain 1 region is not affected by the mutation, but the deletion of domain 3 results in a conformational change in domain 1 , leading to a decrease in overall enzyme function. 


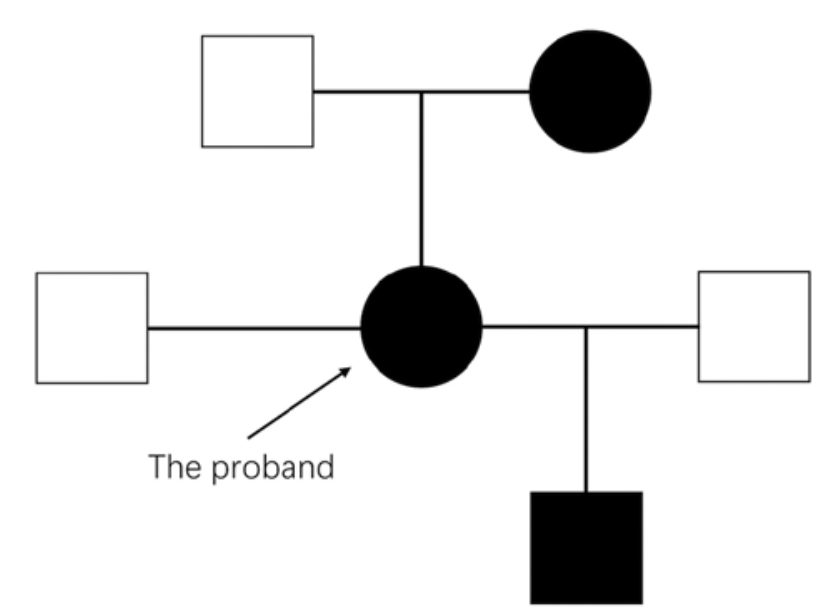

Figure 6. Pedigree chart of the patient's family. Males are indicated by squares and females by circles, and individuals carrying the mutation are indicated in black.

Although PBG and ALA accumulation is hypothesized to be the trigger of AIP (1), their exhaustive pathological role remains elusive. In the present study, the focus was on the neurological manifestations of AIP. Due to the presence of the typical symptoms (headache, visual disturbances and seizures) and characteristic MRI, the patient was clinically diagnosed with PRES.

Brain lesions in patients with acute porphyria are not rare. However, only a few studies have reported on brain MRI features of patients with AIP. MRIs performed early during an attack have demonstrated reversible cortical changes consistent with PRES (14). PRES refers to a reversible subcortical vasogenic brain edema in patients with acute neurological symptoms (15), including seizures, headaches and visual disturbances, which are the three major clinical manifestations of AIP.

Since the first report on PRES in 1996, numerous studies describing the disease have been published, but the exact pathogenesis has remained to be defined. There are currently two widely accepted hypotheses regarding the pathogenesis of PRES: i) Excessive brain perfusion caused by an increase in blood pressure that breaks the cerebral blood flow autoregulation threshold; ii) disruption of the blood-brain barrier(BBB) by vascular endothelial dysfunction due to toxic substances (15). The first theory is the core mechanism leading to PRES, as the posterior cerebrum lacks sympathetic innervation and is sensitive to blood pressure fluctuations (16). Since there was no significant increase in blood pressure in the patient of the present study, it was inferred that PRES was caused by other mechanisms.

Neurovisceral symptoms are one of the typical manifestations of AIP. Autonomic testing has demonstrated parasympathetic and sympathetic dysfunction during an acute attack of AIP (14). Considering the lack of sympathetic innervation in the posterior region of cerebrum, it was concluded that sympathetic dysfunction in AIP was the pathological basis for the initiation of PRES. Parasympathetic, as well as sympathetic dysfunction, makes the arteries in the posterior region more susceptible to vasodilation and hyperperfusion, leading to homeostatic dysregulation of cerebral blood flow (15).
A systematic review reported that AIP complicated with PRES is more common in female patients (17). In the present case, the common causes of PRES, including hypertension and hyponatremia, were not present. It may be hypothesized that pregnancy may be an important cause of AIP attacks and PRES, as it alters hormone secretion, the internal environment and sympathetic function. At the same time, pregnancy may increase the metabolic burden of patients. All of these factors may be the core reasons for the induction of AIP and PRES in the present case.

In fact, through the experiments in the present study, it was confirmed that the mutated HMBS protein had $\sim 60 \%$ activity and the patient's HMBS mutation was heterozygous. This means that under normal circumstances, the HMBS activity in the patient may reach $\sim 80 \%$ of the normal level (50\% contributed from the WT allele and 30\% from the mutant allele). In AIP, however, a 50\% decrease in HMBS activity is typically indicative of the disease (18). Under these conditions, patients usually do not have severe AIP attacks. Therefore, it is hypothesized that the onset of the episode of AIP the present case was associated with a marked increase in hemoglobin demand due to pregnancy. The patient's mutated HMBS was not able to meet the increased hemoglobin synthesis burden, resulting in an abnormal increase in ALA and PBG and leading to this episode of AIP.

The identified HMBS mutation, c.405-406delAA, causes a frameshift and leads to premature termination of the protein. Most likely, this mutation triggers nonsense-mediated decay (NMD) and decreases HMBS mRNA expression, resulting in AIP. NMD is a translation-dependent mRNA surveillance mechanism that helps to maintain the quality of gene expression (19). The function of NMD is to eliminate the production of truncated proteins. A premature termination codon that triggers NMD may give rise to disease by precluding the production of the full-length protein. Therefore, it was speculated that the premature termination of the HMBS protein may trigger NMD, resulting in a decrease in the production of full-length HMBS protein, which in turn induces AIP. This is a potential mechanism for the onset of AIP in this patient.

Abnormal accumulation of ALA and PBG is the major pathological process in AIP (1) and a potential mechanism of PRES (17). ALA is neurotoxic and affects the binding affinity of g-aminobutyric acid (GABA) to its receptors, but not the binding of serotonin or dopamine (14). Studies have indicated that most or all of the ALA and PBG that are toxic to the central nervous system are produced by the metabolism of cells localized in the brain, and the BBB was not, or was only slightly permeable to ALA and PBG $(20,21)$. Hu et al (22) reported that disruption of the 5-ALA transport mechanism is a key factor in ALA neurotoxicity. In the present study, the patient had an AIP attack and PRES. It was speculated that an underlying mechanism affects the permeability of the BBB and induces vasogenic edema in the brain tissue, leading to PRES. At the same time, changes in the permeability of the BBB also lead to changes in the intracranial concentration of ALA, causing neurotoxicity of ALA.

Inhibition of GABA receptors increased vascular endothelial growth factor (VEGF) expression in tumor cells (23). VEGF is an important mediator of vascular permeability, and changes in BBB permeability due to changes in VEGF 
expression are also important causes of PRES (24). Increased levels of circulating VEGF induces brain edema seen in PRES (24). Thus, it was speculated that accumulating ALA may act on GABA receptors of astrocytes and alter VEGF expression. This possible mechanism has remained to be confirmed by experimental studies and will require further exploration in the future.

ALA also reversibly inhibits energy-dependent $\mathrm{Na}^{+} / \mathrm{K}^{+}$ ATPase in the brain, which may subsequently lead to the development of cellular edema (14). In addition, the production of nitric oxide (NO) may also decrease during the onset of AIP and cause vasoconstriction (25). NO synthase is a hemoprotein and the relative deficiency of heme during AIP may reduce the production of NO (26).

Currently, there are no established criteria and guidelines for the diagnosis and classification of PRES. Although not $100 \%$ specific, imaging findings combined with clinical symptoms are acceptable for the diagnosis of PRES. The T2-weighted imaging (T2WI) or T2-fluid-attenuated inversion recovery (FLAIR) hyperintensities in the posterior region of the brain is a typical imaging pattern of PRES, which indicates the manifestation of vasogenic edema, observed in $>95 \%$ of patients with PRES (16). However, cytotoxic edema is also observed on diffusion-weighted imaging in certain patients (27). In the case of the present study, T2WI or T2-FLAIR hyperintensities were detected in the posterior region of the brain and significant diffusion restriction was observed, indicating vasogenic and cytotoxic edema in this patient. These results confirmed the inference that PRES in this patient was mainly due to toxic metabolites. It is noteworthy to mention that the presence of extensive vasogenic edema or diffusion restriction is associated with an unfavorable clinical outcome (28). However, contrary to certain studies (16), the patient of the present study recovered completely. It may therefore be hypothesized that lesions caused by restricted diffusion may not be irreversible.

AIP diagnosis during or following an acute attack is usually based on clinical symptoms combined with PBG excretion. Over the years, genetic analysis has become the most reliable method to confirm AIP in symptomatic patients, and asymptomatic patients who do not always have elevated levels of ALA and PBG, as well as in patients with the non-erythroid variant of AIP with a normal level of urinary ALA and PBG (2).

There are several limitations to the present study. First, due to the experimental limitations, results on the ALA concentration in patient samples could not be obtained. The ALA concentration is considered necessary for the diagnosis of AIP. However, AIP was diagnosed by the Watson-Schwartz test, which has been proven feasible in the diagnosis of AIP. In addition, it was not possible to isolate RNA from the patient to assess HMBS mRNA expression levels, although it was speculated that NMD-induced mRNA degradation of HMBS may be a potential pathogenesis of AIP. Complementary experiments will be performed in future studies to clarify the pathogenesis associated with the mutant HMBS.

\section{Acknowledgements}

Not applicable.

\section{Funding}

No funding was received.

\section{Availability of data and materials}

The datasets used and/or analyzed during the current study are available from the corresponding author on reasonable request.

\section{Authors' contributions}

YY, XC and ZY contributed to the conception and design of the study. HW and HP provided important medical decisions in the clinical treatment of patient, contributed the acquisition and analysis of clinical data and wrote the Case Report section. WS and $\mathrm{BH}$ performed the experimental study. YY and XC wrote the first draft of the manuscript. $\mathrm{ZY}$ and $\mathrm{BH}$ wrote sections of the manuscript. All authors contributed to manuscript revision and they read and approved the submitted version.

\section{Ethics approval and consent to participate}

All procedures followed were in accordance with the ethical standards of the Ethics Committee of The Second Military Medical University, and with The Helsinki Declaration from 1975 and its revision from 2000. The patient provided informed consent for genetic testing and the study was approved by the ethics committee of Second Military Medical University (Shanghai, China).

\section{Patient consent for publication}

The patient, her parents and her child provided informed consent for publication of this manuscript.

\section{Competing interests}

The authors declare that they have no competing interests.

\section{References}

1. Stölzel U, Doss MO and Schuppan D: Clinical guide and update on porphyrias. Gastroenterology 157: 365-381.e4, 2019.

2. Pischik E and Kauppinen R: An update of clinical management of acute intermittent porphyria. Appl Clin Genet 8: 201-214, 2015.

3. Edel Y and Mamet R: Porphyria: What is it and who should be evaluated? Rambam Maimonides Med J 9, 2018.

4. Walterfang M, Bonnot O, Mocellin R and Velakoulis D: The neuropsychiatry of inborn errors of metabolism. J Inherit Metab Dis 36: 687-702, 2013.

5. Phillips JD: Heme biosynthesis and the porphyrias. Mol Genet Metab 128: 164-177, 2019.

6. Vilas-Boas S and Corte-Real A: Posterior reversible encephalopathy syndrome and azathioprine. Eur J Case Rep Intern Med: 6: 001032, 2019.

7. De Siervi A, Rossetti MV, Parera VE, Astrin KH, Aizencang GI, Glass IA, Batlle AM and Desnick RJ: Identification and characterization of hydroxymethylbilane synthase mutations causing acute intermittent porphyria: Evidence for an ancestral founder of the common G111R mutation. Am J Med Genet 86: 366-375, 1999.

8. Sun G, Wang C, Zhen J, Zhang G, Xu Y and Deng Z: Cloning and sequencing of KIR2DL1 Framework Gene cDNA and identification of a novel allele. Zhonghua Yi Xue Yi Chuan Xue Za Zhi 33: 694-697, 2016 (In Chinese). 
9. Unzu C, Sampedro A, Mauleón I, Alegre M, Beattie SG, de Salamanca RE, Snapper J, Twisk J, Petry H, González-Aseguinolaza G, et al: Sustained enzymatic correction by rAAV-mediated liver gene therapy protects against induced motor neuropathy in acute porphyria mice. Mol Ther 19: 243-250, 2011.

10. Chen B, Solis-Villa C, Erwin AL, Balwani M, Nazarenko I, Phillips JD, Desnick RJ and Yasuda M: Identification and characterization of 40 novel hydroxymethylbilane synthase mutations that cause acute intermittent porphyria. J Inherit Metab Dis 42: 186-194, 2019.

11. Corbett MB: The Watson-Schwartz Test. JAMA 195: 481, 1966.

12. Elder GH and Sandberg S: Identifying acute porphyria in patients with acute polyneuropathy or encephalopathy. Nat Clin Pract Neurol 4: 648-649, 2008.

13. Gill R, Kolstoe SE, Mohammed F, Al D-Bass A, Mosely JE, Sarwar M, Cooper JB, Wood SP and Shoolingin-Jordan PM: Structure of human porphobilinogen deaminase at $2.8 \mathrm{~A}$ : The molecular basis of acute intermittent porphyria. Biochem J 420 : $17-25,2009$.

14. Simon NG and Herkes GK: The neurologic manifestations of the acute porphyrias. J Clin Neurosci 18: 1147-1153, 2011.

15. Fugate JE and RabinsteinAA: Posterior reversible encephalopathy syndrome: Clinical and radiological manifestations, pathophysiology, and outstanding questions. Lancet Neurol 14: 914-925, 2015.

16. Ollivier M, Bertrand A, Clarençon F, Gerber S, Deltour S, Domont F, Trunet S, Dormont D and Leclercq D: Neuroimaging features in posterior reversible encephalopathy syndrome: A pictorial review. J Neurol Sci 373: 188-200, 2017.

17. Zheng X, Liu X, Wang Y, Zhao R, Qu L, Pei H, Tuo M, Zhang Y, Song Y, Ji X, et al: Acute intermittent porphyria presenting with seizures and posterior reversible encephalopathy syndrome: Two case reports and a literature review. Medicine (Baltimore) 97: e11665, 2018

18. Arora S, Young S, Kodali S and Singal AK: Hepatic porphyria: A narrative review. Indian J Gastroenterol 35: 405-418, 2016.

19. Kurosaki T and Maquat LE: Nonsense-mediated mRNA decay in humans at a glance. J Cell Sci 129: 461-467, 2016
20. Yasuda M, Gan L, Chen B, Yu C, Zhang J, Gama-Sosa MA, Pollak DD, Berger S, Phillips JD, Edelmann W and Desnick RJ: Homozygous hydroxymethylbilane synthase knock-in mice provide pathogenic insights into the severe neurological impairments present in human homozygous dominant acute intermittent porphyria. Hum Mol Genet 28: 1755-1767, 2019.

21. Solis C, Martinez-Bermejo A, Naidich TP, Kaufmann WE, Astrin KH, Bishop DF and Desnick RJ: Acute intermittent porphyria: studies of the severe homozygous dominant disease provides insights into the neurologic attacks in acute porphyrias. Arch Neurol 61: 1764-1770, 2004.

22. Hu Y, Shen H, Keep RF and Smith DE: Peptide transporter 2 (PEPT2) expression in brain protects against 5-aminolevulinic acid neurotoxicity. J Neurochem 103: 2058-2065, 2007.

23. Fava G, Marucci L, Glaser S, Francis H, De Morrow S, Benedetti A, Alvaro D, Venter J, Meininger C, Patel T, et al: gamma-Aminobutyric acid inhibits cholangiocarcinoma growth by cyclic AMP-dependent regulation of the protein kinase A/extracellular signal-regulated kinase 1/2 pathway. Cancer Res 65: 11437-11446, 2005.

24. Chen Z, Shen GQ, Lerner A and Gao B: Immune system activation in the pathogenesis of posterior reversible encephalopathy syndrome. Brain Res Bull 131: 93-99, 2017.

25. Olivier P, Van Melkebeke D, Honoré PJ, Defreyne L and Hemelsoet D: Cerebral vasospasm in acute porphyria. Eur J Neurol 24: 1183-1187, 2017.

26. Thachil J: Nitric oxide and the clinical manifestations of acute porphyria. Intern Med J 38: 732-735, 2008.

27. Gao B, Lyu C, Lerner A and McKinney AM: Controversy of posterior reversible encephalopathy syndrome: What have we learnt in the last 20 years? J Neurol Neurosurg Psychiatry 89: $14-20,2018$

28. Schweitzer AD, Parikh NS, Askin G, Nemade A, Lyo J, Karimi S, Knobel A, Navi BB, Young RJ and Gupta A: Imaging characteristics associated with clinical outcomes in posterior reversible encephalopathy syndrome. Neuroradiology 59: 379-386, 2017.

(i) $\ominus$ This work is licensed under a Creative Commons

cc) ${ }_{\mathrm{EY}}$ NO ND Attribution-NonCommercial-NoDerivatives 4.0 International (CC BY-NC-ND 4.0) License. 